\title{
Patient Absconding and Its Predictors: A 5-Year Retrospective Analysis in a General Teaching Hospital in Tehran, Iran
}

\author{
Somayeh Anisi, ${ }^{1}$ Ehsan Zarei, ${ }^{2,}{ }^{*}$ Hamid Kariman, ${ }^{3}$ Mohammad Kazem Kazemi, ${ }^{1}$ and Mohammad \\ Chehrazi $^{4}$ \\ ${ }^{1}$ MSc, Health Services Management, Imam Hussein Hospital, Shahid Beheshti University of Medical Science, Tehran, IR Iran \\ ${ }^{2} \mathrm{PhD}$ in Health Service Management, Department Of Public Health, Faculty of Health, Shahid Beheshti University of Medical Sciences, Tehran, IR Iran \\ ${ }^{3}$ Associate Professor in Emergency Medicine, Imam Hussein Hospital, Shahid Beheshti University of Medical Sciences, Tehran, IR Iran \\ ${ }^{4} \mathrm{PhD}$ in Biostatistics, Department of Epidemiology and Reproductive Health, Reproductive Epidemiology Research Center, Royan Institute for Reproductive Biomedicine, \\ ACECR, Tehran, Iran
}

"Corresponding author: Ehsan Zarei, Department Of Public Health, Faculty of Health, Shahid Beheshti University of Medical Sciences, Daneshjo Blvd., Velenjak, Tehran, IR Iran. Tel: +98-2122432040-41, E-mail: e.zarei@sbmu.ac.ir

Received 2017 June 06; Revised 2017 July 07; Accepted 2017 September 11.

\begin{abstract}
Background and Purpose: Patient absconding from a hospital or leaving without permission is a major challenge with economic and social consequences for patients, healthcare staff, and society. Identification of the characteristics of absconding patients is essential to the prediction and prevention of such behaviors.

Methods: This cross sectional study was performed in a large general teaching hospital in Tehran, Iran. Data of 460 absconding patients and 460 nonabsconding patients, admitted during March 2010 - March 2016, were extracted from the hospital information system. Backward stepwise logistic regression analysis was used to describe the importance and effect of each predictor.

Results: The findings showed that $0.4 \%$ of the patients had left the hospital without permission or notice to the staff. Addiction (OR, 27.15), lack of insurance coverage (OR, 12.39), male gender (OR, 2.14), age $\leq 40$ years (OR, 1.89), and emergency admission (OR, 1.77) were predictors of absconding. Hospital financial loss amounted to $3,818,788,624 \operatorname{IRR}(\$ 126,283)$ due to this problem, with an average cost of 7,178,174 IRR (\$237) per patient.

Conclusions: Identification of high-risk individuals, including young men, uninsured patients, and drug addicts with emergency admission warrants preventive measures. Moreover, assessment of patients by physicians or nurses upon admission, staff training and raising awareness, and use of technologies such as radiofrequency identification can be other strategies for preventing or reducing this problem.
\end{abstract}

Keywords: Patient Absconding, General Hospital, Inpatient

\section{Background}

Patient absconding from hospital without permission is a serious health issue with financial, social, and health implications (1). Patient absconding is defined as leaving a medical center without permission from service providers (2), which can pose risks to patients, their families, and hospital staff. The potential consequences of patient absconding include self-harm, suicide, violence, harm to others, self-neglect, death, treatment disruption, lengthened recovery, and stigmatized reputation of the hospital and health system (3-6).

The national quality forum in the United States considers patient absconding as a serious problem (7). Patient absconding can lead to an increase in the workload of hospital staff and security and add to the required measures for returning the patient (8). Hospitals, as well as the staff, may also encounter legal problems in case of harm to the patients (9). Financial loss due to unpaid bills (1) and being sued by patients' families are among the other consequences of patient absconding (10). Moreover, this issue may have emotional implications, such as fear, worry, and guilt, besides legal matters such as legal charges by colleagues or authorities, penalties, and judicial summons (11).

The rate of patient absconding in various studies has been estimated at $2.5 \%-34 \%$ in psychiatric hospitals (12) and $0.27 \%-2.4 \%$ in general hospitals $(1,3,13,14)$. Studies have shown that absconding is common among young individuals, men, drug addicts, patients admitted to emergency departments (ED), and those with imprisonment experiences $(3,12-17)$. In various studies, it has been shown that absconding occurs more frequently during warm seasons. In a review study, most absconding occurred on weekends, whereas other studies have not specified such patterns (18). 
The causes of patient absconding include fear, homesickness, irritation, concerns about family responsibilities, delayed medical examinations and treatments, treatment costs, lack of insurance coverage, lack of information about the disease, and mental disorders (3, 15, 17-19). According to a study by Handel et al., reducing the waiting time for patients and informing them about the approximate waiting time can dissipate the number of escapes (20).

According to the joint commission on accreditation of healthcare organizations (JCAHO), the primary contributors to patient absconding are failure in patient assessment and team communication (9). Risk assessment of absconding upon admission and during the patient's stay has been noted in various studies for reducing and preventing absconding and improving patient safety (21, 22). Guidelines and instructions need to be compiled and applied in risk estimation of absconding, and strategies should be developed for reducing and preventing absconding in high-risk patients (22).

Regarding the importance of economic, social, and medical implications of absconding for hospitals, prevention can contribute to a decline in the resulting damage. Hospitals are also required to take effective measures for preventing patient absconding. Therefore, investigation of patient absconding provides important information on the causes of absconding and introduces effective strategies to reduce this behavior (3). Few studies have been performed on patient absconding in general hospitals, mainly focusing on the characteristics of patient absconding rather than analyzing the predictive factors. However, the duration of these studies is limited, and therefore, they fail to provide comprehensive data $(13,14)$. In this study, we investigated the characteristics of patient absconding and its predictors during 5 years in a large general hospital in Tehran, Iran.

\section{Methods}

This retrospective study was based on the electronic medical records of all patients, who were admitted to a large general hospital in Tehran during 2011 - 2016 (Mar 21, 2011-Mar 20, 2016). This hospital is a national teaching referral center with more than 500 beds. During the study, 535 absconding patients were reported in the hospital, 75 of whom were excluded due to information deficiency. Finally, the study sample consisted of 460 absconding patients and 460 discharged patients as the control group.

The control group consisted of inpatients, who had been discharged from the hospital as planned. Each control subject was matched in terms of time (year) with a case (absconding) subject, and the samples were selected through random sampling. The data were extracted from the hospital information system (HIS), using Crystal Reports software. In case of deficiency in the data, recorded reports, such as the supervisor's reports or medical records, were used to complete the data.

The variables of age, gender, marital status, place of residence, insurance coverage, length of stay (LOS), type of admission (emergency or elective), weekdays, work shifts, and patient's out-of-pocket payment were considered as the independent variables, while patient absconding was regarded as the dependent variable. To compare the variables in the groups of absconding and nonabsconding patients, independent two-sample t test was applied for quantitative variables, while Chi square test was used for qualitative variables.

Backward stepwise logistic regression analysis was used to describe the importance and effect of each predictor of absconding on the variables in the model. The relationship between variables was assessed using odds ratio (OR) and 95\% confidence interval (CI). SPSS version 21 was used to analyze the data. According to the Central Bank of Iran, the value of 1 USD was 30,240 Iranian Rials (IRR) in 2016 (23). To collect the data, coordination was made with the hospital managers, and confidentiality of the patients' data was respected throughout the study. This study was approved by the research ethics committee of Shahid Beheshti University of Medical Sciences (code, SBMU.RETECH.REC.1395.453).

\section{Results}

Among 132,375 admitted patients in 5 years, 535 patients had left the hospital without permission or informing the staff, and the patient absconding rate was $0.4 \%$ in the hospital. Out of every 250 hospital admissions, one case of absconding had occurred. The hospital financial loss amounted to $3,818,788,624$ IRR (\$126 283) due to this problem, with an average cost of 7,178,174 IRR (\$237) per patient.

The average age of patients was $35( \pm 15.5)$ years in the absconding group and $47( \pm 21.4)$ years in the nonabsconding group $(\mathrm{P}<0.001$; Table 1$)$. Over two-thirds of absconding patients (68.7\%) were younger than 40 years. Frequency of hospitalization was 1.69 among absconding patients and 5.71 among nonabsconding patients $(\mathrm{P}<0.001)$. Nearly $75 \%$ of absconding patients did not have a history of hospitalization. The LOS was $6( \pm 9.5)$ days among absconding patients and 5.7 ( \pm 8.4 ) days among nonabsconding patients $(\mathrm{P}=0.603)$.

As presented in Table 2, Chi square test results indicated a significant difference between the absconding and 
Table 1. Comparison of Age, History of Hospitalization, and LOS in Absconding and Nonabsconding Patients ${ }^{\mathrm{a}}$

\begin{tabular}{lccc}
\hline Variables & $\begin{array}{c}\text { Absconding } \\
\text { Patients }\end{array}$ & $\begin{array}{c}\text { Nonabsconding } \\
\text { Patients }\end{array}$ & P Value \\
\hline Age & $35.60 \pm 15.48$ & $47.04 \pm 21.36$ & $<0.001$ \\
LOS & $6.02 \pm 9.47$ & $5.71 \pm 8.39$ & 0.603 \\
$\begin{array}{l}\text { History of } \\
\text { hospitalization }\end{array}$ & $1.69 \pm 5.63$ & $5.29 \pm 10.33$ & $<0.001$ \\
\hline
\end{tabular}

Abbreviation: SD, Standard deviation.

${ }^{\mathrm{a}}$ Values are expressed as mean $\pm \mathrm{SD}$.

nonabsconding patients in terms of gender, marital status, place of residence, admission type, and insurance coverage $(\mathrm{P}<0.001)$. Among absconding patients, $78.3 \%(\mathrm{n}, 360)$ were male and $45.4 \%$ were single. Overall, $57.8 \%$ (n, 266) of absconding patients lived in Tehran, and 13.7\% had no address. Most absconding patients ( $85.4 \%$ ) were admitted to ED, and 92.4\% (n, 425) were uninsured.

The lowest rate of absconding (n,36; 7.8\%) was reported on weekends (Friday in Iran), while the highest rate (n, $76 ; 16.5 \%$ ) was reported on Saturdays (first day of the week in Iran). However, there was no significant difference between days of week $(\mathrm{P}=0.83)$. The highest rate of absconding $(n, 130 ; 28.3 \%)$ occurred in summer, while the lowest rate $(n, 104 ; 22.6 \%)$ was reported in autumn; however, there was no significant difference among the seasons $(\mathrm{P}=0.45)$. The results showed that most absconding occurred during morning shifts (73.9\%), while the lowest rate was reported during night shifts $(\mathrm{P}<0.001)$. Moreover, the highest rate of absconding was found in the surgical ward (25.4\%), internal ward (17.4\%), and orthopedic ward (16.3\%) $(\mathrm{P}<0.001)$.

Based on the findings, cardiac surgery intensive care unit (CSICU), pediatric ward, psychiatric ward, and ICU did not have any absconding patients. In terms of primary diagnosis of patients based on the international classification of diseases (ICD), the most common diagnosis was trauma among absconding patients (code S00-T98;167 cases) and circulatory systemic diseases in nonabsconding patients (code I00-I99; 74 cases). Overall, 36.3\% (n, 167) of absconding and $13.7 \%$ ( $n, 63)$ of nonabsconding patients were admitted to the hospital due to an external trauma and injury.

The most common cause of trauma among absconding patients was injury by a sharp or blunt object (41.6\%; codes X99, Y28, and Y29), while for nonabsconding patients, it was traffic accident (44.6\%; codes Voo-V99); the cause of trauma in 2 patients was suicide (codes X78- X79). There was a significant difference between the groups in terms of primary diagnosis $(\mathrm{P}<0.001)$. The findings indicated that $13.3 \%(n, 61)$ of absconding and $0.7 \%(n, 3)$ of nonabscond- ing patients were addicted to drugs $(\mathrm{P}<0.001)$.

The results of logistic regression analysis showed that variables, including addiction to drugs (OR, 27.15), lack of insurance coverage (OR, 12.39), gender (OR, 2.14), age (OR, $1.89)$, and type of admission (OR, 1.77) were predictive factors for patient absconding. Based on these results, the most important predictive factor was addiction; in fact, the OR of absconding in addicted patients was 27 times higher than other patients. Uninsured patients absconded more than insured patients, and the OR of absconding was 2.14 times higher in men compared to women. In addition, the OR of absconding in younger patients ( $\leq 40$ years) was twice as high as other patients. Patients who had been admitted to ED tended to abscond more than other patients (Table 3).

\section{Discussion}

This study investigated the characteristics of absconding patients and examined the predictors of absconding in a large general teaching hospital in Tehran, Iran. The results showed that the absconding rate was $0.4 \%$, which is similar to previous studies. Similar rates have been reported in studies performed in Shiraz (0.5\%) (8), Tehran (1.0\%) (14), and Hong Kong (0.27\%) (13). Despite the low prevalence of absconding, this behavior should be considered by hospital and health system authorities, given its economic, social, legal, and medical implications.

Based on the findings, the OR of absconding in male patients was 2.1 times higher than females, which is similar to the findings of previous studies. In studies by Khemernia et al. and Gerace et al. the OR of absconding in male patients was 1.37 and 2 times higher than female patients, respectively $(3,24)$. Moreover, the findings of a review study indicated that the risk of absconding in men was higher than women (18). In other studies, the escape rate in male patients was higher than female patients $(1$, 13, 14). The differences in decision-making mechanisms between men and women, as well as women's lower risktaking and more sensation-seeking behaviors (25), can contribute to the lower rate of absconding among women.

In the present study, the risk of absconding in younger patients was higher than older patients, which is similar to previous studies $(1,8,14,26)$. In this regard, in a review study, absconding patients were younger than others (18). In fact, younger people have relatively more rebellious and adventurous behaviors (27), which can bring about a stronger motive for absconding. Medical insurance coverage was the second predictor of absconding, and the risk of absconding in uninsured patients was 12 times higher than that of insured patients. In some studies performed 
Table 2. Comparison of Demographic Variables in Absconding and Nonabsconding Patients ${ }^{\mathrm{a}}$

\begin{tabular}{|c|c|c|c|c|}
\hline Variables & & Absconding Patients & Nonabsconding Patients & P Value \\
\hline \multirow{2}{*}{ Gender } & Male & $360(78.3)$ & $246(53.5)$ & $<0.001$ \\
\hline & Female & $100(21.7)$ & $214(46.5)$ & \\
\hline \multirow{3}{*}{ Marital status } & Single & $203(44.1)$ & $114(24.8)$ & $<0.001$ \\
\hline & Married & $251(54.6)$ & $341(74.1)$ & \\
\hline & Divorced & $6(1.3)$ & $5(1.1)$ & \\
\hline \multirow{3}{*}{ Place of residence } & Tehran & $266(57.8)$ & $285(62)$ & $<0.001$ \\
\hline & Other cities & $131(28.5)$ & $166(36.1)$ & \\
\hline & No address & $63(13.7)$ & $9(2)$ & \\
\hline \multirow{2}{*}{ Admission type } & Emergency & $393(85.4)$ & $262(57)$ & $<0.001$ \\
\hline & Elective & $67(14.6)$ & $198(43)$ & \\
\hline \multirow{2}{*}{ Insurance coverage } & Insured & $35(7.6)$ & $273(59.3)$ & $<0.001$ \\
\hline & Uninsured & $425(92.4)$ & $187(40.7)$ & \\
\hline
\end{tabular}

${ }^{\mathrm{a}}$ Values are expressed as No. (\%).

Table 3. Predictive Variables for Patient Absconding Based on the Logistic Regression Analysis

\begin{tabular}{|c|c|c|c|c|}
\hline \multirow[t]{2}{*}{ Variables $^{\mathrm{a}}$} & \multirow[t]{2}{*}{ Odds Ratio (OR) } & \multicolumn{2}{|c|}{ 95\% CI for $\mathrm{OR}^{\mathrm{b}}$} & \multirow[t]{2}{*}{ P Value } \\
\hline & & Low & High & \\
\hline Drug addiction (addicted/not addicted) & 27.15 & 8.14 & 90.53 & $<0.001$ \\
\hline Insurance (uninsured/insured) & 12.39 & 8.14 & 18.87 & $<0.001$ \\
\hline Gender (male/female) & 2.14 & 1.49 & 3.07 & $<0.001$ \\
\hline Age, $\leq \mathbf{4 0} />40 \mathrm{y}$ & 1.89 & 1.33 & 2.68 & $<0.001$ \\
\hline Admission type (emergency/elective) & 1.77 & 1.21 & 2.59 & 0.003 \\
\hline
\end{tabular}

Abbreviation: $\mathrm{CI}$, confidence interval.

${ }^{a}$ Each secondary variable was considered as the reference variable (eg, female is the reference for the variable of gender)

${ }^{\mathrm{b}}$ Significance level at 0.05

in Iran, 60\% - 70\% of absconding patients did not have insurance coverage $(1,3,14)$. The findings of a study in Shiraz, Iran also indicated that lack of insurance coverage was the strongest predictor of patient absconding, which is consistent with our findings (3).

Treatment cost is one of the main reasons for patient absconding, as mentioned in previous studies $(3,28)$. In fact, patients and their families cannot afford the treatment costs and leave the hospital without informing the physician or medical staff. Moreover, the findings of the present study indicated that most absconding patients (85.4\%) were admitted to the ED, and the risk of absconding in these patients was 1.8 times higher than others. Moreover, the findings of a study by Cheng et al. indicated that $95.7 \%$ of absconding patients were admitted to $\operatorname{ED}(13)$, which is in line with our study. It is possible to justify this finding by the patients' primary diagnosis. Most of these patients (36\%) were admitted to the hospital because of a traumatic event (traffic accident or fight) and might have escaped for fear of legal consequences.

In another study, the majority of traumas among absconders were caused by a sharp or blunt object (knife) unintentionally or in an assault (14). The American College of emergency physicians (ACEP) identifies avoidance of contact with statutory agencies as a probable cause of absconding in patients with injuries (15). Regarding the fact that the studied hospital was a trauma center, it is necessary to deploy police forces and implement strict inspection by security guards. Moreover, interactions with the clinical staff for identifying and caring for patients, who are prone to absconding, can be effective in reducing absconding.

Drug use appears to be one of the reasons for disease because of the signs of drug deprivation during admission, 
impaired judgment, and disruptions in the treatment process. The findings of this study indicated that $13.3 \%$ of absconding patients were addicted to drugs, and the risk of absconding in these patients was 27 times higher than others. A study in Tehran indicated that $19.5 \%$ of absconding patients were drug addicts (14), which is consistent with our study. It seems that drug abuse alone brings about symptoms, such as withdrawal syndrome and judgment disorders during hospitalization, and cause disturbances in the treatment process, which can be a reason for patient absconding.

On the other hand, the multifaceted relationship between drug use, other individual characteristics, and social harm should be considered. Drug abuse is recognized as a major predisposing factor for almost all types of trauma. In previous studies, the association between drug abuse, violence, aggressive and threatening behaviors, consequent traumas, and traffic accidents has been clarified $(29,30)$. Drug abuse leads to violence and plays a crucial role in criminal behaviors. Based on comparisons, patients with a trauma or injury history are exposed to a greater risk of drug abuse (30).

In a study by Soroush et al. drug abuse was reported in $27 \%$ of patients with trauma (31). Fear of prosecution by authorities and victimization are among the reasons for patient absconding. In addition, the bilateral association between addiction, poverty, unemployment, and lack of insurance coverage can show that patients are unable to afford their treatment costs, thus leading to patient absconding. It seems impossible to construe absconding-related factors alone or regardless of their affiliation with one another. This phenomenon is a consequence of the interaction between social and individual characteristics, which merely indicates a cause-and-effect relationship between absconding and a particular characteristic.

One limitation of the present study is the incompleteness of data in HIS and medical records. In addition, in this study, reports on drug abuse were used, which might have been underestimated due to lack of patient information or history in the medical records.

\subsection{Conclusions}

Despite the low rate of patient absconding in general hospitals and the social, economic, and legal implications of this phenomenon on patients, hospitals, and health system, prevention and reduction measures seem necessary. Identification of patients at high risk of absconding, such as young individuals, men, uninsured patients, addicts, and ED-admitted patients, can be beneficial for exercising more care and taking preventive measures. Other ways of preventing and reducing patient absconding include patient risk assessment on admission, educating and informing the staff, use of technologies such as radiofrequency identification (RFID), paying attention to the patients' needs, and cooperation between the patients' families and their caregivers.

\section{Footnote}

Funding/Support: Shahid Beheshti University of Medical Science.

\section{References}

1. Zarei E, Marzban S, Rajaee R, Najafi M. Patient absconding from emergency department and its financial burden: a study in a large public hospital, Tehran. J Health Field. 2017;3(1).

2. Reddy KVR, Kumar GS, Vijayalakshmi D. A Study on Reasons of Absconds From Inpatient Ward in Government Hospital for Mental Care Visakhapatnam. Bipolar Disord. ;70:30.17.

3. Khammarnia M, Kassani A, Amiresmaili M, Sadeghi A, Karimi Jaberi Z, Kavosi Z. Study of patients absconding behavior in a general hospital at southern region of Iran. Int J Health Policy Manag. 2015;4(3):137-41. doi: 10.15171/ijhpm.2014.110. [PubMed: 25774367].

4. Cullen AE, Jewell A, Tully J, Coghlan S, Dean K, Fahy T. A Prospective Cohort Study of Absconsion Incidents in Forensic Psychiatric Settings: Can We Identify Those at High-Risk? PLoS One. 2015;10(9):e0138819. doi: 10.1371/journal.pone.0138819. [PubMed: 26401653].

5. Dickens GL, Campbell J. Absconding of patients from an independent UK psychiatric hospital: a 3-year retrospective analysis of events and characteristics of absconders. J Psychiatr Ment Health Nurs. 2001;8(6):543-50. [PubMed: 11842482].

6. Hunt IM, Clements C, Saini P, Rahman MS, Shaw J, Appleby L, et al. Suicide after absconding from inpatient care in England: an exploration of mental health professionals' experiences. $J$ Ment Health. 2016;25(3):245-53. doi: 10.3109/09638237.2015.1124394. [PubMed: 27150467].

7. National Quality Forum. Serious reportable events in healthcare 2017. Available from: http//www.qualityforum.org/Publications/2008/10/ Serious_Reportable_Events.aspx.

8. Khammarnia M, Kavosi Z, Karimi Jaberi Z, Shirvani M, Sadeghi A. Investigation of the rate of patients escape from a general hospital in Shiraz, Iran. Sadra Med Sci J. 2014;2(1 Jan).

9. Digiarts . Tyco integrated security.9 ways to help deter patient elopement.Tyco integrated security 2017. Available from: http://www.digiarts.org/tycohealthcaresecurity.com/pdf/ADT_ whitepaper_elopemnt.pdf.

10. Smith TA. Hospital security and patient elopement: protecting patients and your healthcare facility. J Healthc Prot Manage. 2012;28(1):720. [PubMed: 22423516].

11. Grotto J, Gerace A, O'Kane D, Simpson A, Oster C, Muir-Cochrane E. Risk assessment and absconding: perceptions, understandings and responses of mental health nurses. J Clin Nurs. 2015;24(5-6):855-65. doi: 10.1111/jocn.12671. [PubMed: 25209549].

12. Muir-Cochrane E, Mosel KA. Absconding: A review of the literature 1996-2008. Int J Ment Health Nurs. 2008;17(5):370-8. doi: 10.1111/j.14470349.2008.00562.x. [PubMed: 18789047].

13. Cheng ST, Chung CH, Leung YH, Lai KK. Patient absconding behaviour in a public general hospital: retrospective study. Hong Kong Med J. 2002;8(2):87-91. [PubMed: 11937662].

14. Memarian A, Yazdinezhad SR, Mehrpisheh S, Aghakhani K. Characteristics of absconders from a general health service, Rasoul Akram Hospital in 2013. Pol Ann Med. 2015;22(2):82-5. doi: 10.1016/j.poamed.2015.04.011. 
15. James F, Catherine H. CEC Best Practice guideline-The Patient who Absconds. London: The College of Emergency Medicine; 2013.

16. Kasmi Y, Brennan D. Developing a patient leave request form for a secure setting. Ment Health Pract. 2015;18(5):30-5. doi: 10.7748/mhp.18.5.30.e907.

17. Wilkie T, Penney SR, Fernane S, Simpson AI. Characteristics and motivations of absconders from forensic mental health services: a casecontrol study. BMC Psychiatry. 2014;14:91. doi: 10.1186/1471-244X-14-91. [PubMed: 24669758].

18. Stewart D, Bowers L. Absconding from psychiatric hospitals: a literature review. London: Institute of Psychiatry, King's College London; 2010.

19. Simpson AI, Penney SR, Fernane S, Wilkie T. The impact of structured decision making on absconding by forensic psychiatric patients: results from an A-B design study. BMC Psychiatry. 2015;15:103. doi: 10.1186/s12888-015-0474-1. [PubMed: 25935745].

20. Handel DA, Fu R, Daya M, York J, Larson E, John McConnell K. The use of scripting at triage and its impact on elopements. Acad Emerg Med. 2010;17(5):495-500. doi: 10.1111/j.1553-2712.2010.00721.x. [PubMed: 20536803].

21. Agency for Healthcare Research and Quality . Elopement 2017. Available from: https://psnet.ahrq.gov/webmm/case/164.

22. Rowe $M$. Wandering in hospitalized older adults: identifying risk is the first step in this approach to preventing wandering in patients with dementia. Am J Nurs. 2008;108(10):62-70. doi: 10.1097/01.NAJ.0000336968.32462.c9. [PubMed:18827546] quiz 71.

23. Central Bank Of The Islamic Republic Of IRAN . Foreign Exchange Rates 2017. Available from: http://www.cbi.ir/exrates/rates_fa.aspx.

24. Gerace A, Oster C, Mosel K, O'Kane D, Ash D, Muir-Cochrane E.
Five-year review of absconding in three acute psychiatric inpatient wards in Australia. Int J Ment Health Nurs. 2015;24(1):28-37. doi: 10.1111/inm.12100. [PubMed: 25444670].

25. Fattore L. Reward processing and drug addiction: does sex matter? Front Neurosci. 2015;9:329. doi: 10.3389/fnins.2015.00329. [PubMed: 26483620].

26. Meehan T, Morrison P, McDougall S. Absconding behaviour: an exploratory investigation in an acute inpatient unit. Aust N Z J Psychiatry. 1999;33(4):533-7. doi: 10.1080/j.1440-1614.1999.00603.x. [PubMed: 10483848].

27. Maddah M, Kheirkhahan E. Effective factors on the commitment of crimes (theft and drug-related). Q Crim Intelligence Res. 2015.

28. Khisty N, Raval N, Dhadphale M, Kale K, Javadekar A. A prospective study of patients absconding from a general hospital psychiatry unit in a developing country. J Psychiatr Ment Health Nurs. 2008;15(6):45864. doi: 10.1111/j.1365-2850.2008.01249.x. [PubMed: 18638205].

29. Khanjani N, Mousavi M, Dehghanian A, Jahani Y, Souri H. The role of drug and alcohol use and the risk of motor vehicle crashes in Shiraz, Iran, 2014: A case-crossover study. Traffic Inj Prev. 2017;18(6):573-6. doi: 10.1080/15389588.2017.1279736. [PubMed: 28095031].

30. Sommer J, Hinsberger M, Elbert T, Holtzhausen L, Kaminer D, Seedat $\mathrm{S}$, et al. The interplay between trauma, substance abuse and appetitive aggression and its relation to criminal activity among high-risk males in South Africa. Addict Behav. 2017;64:29-34. doi: 10.1016/j.addbeh.2016.08.008. [PubMed: 27540760].

31. Soroush AR, Modaghegh MHS, Karbakhsh M, Zarei MR. Drug abuse in hospitalized trauma patients in a university trauma care center: an explorative study. Tehran Univ Med JTUMS Pub. 2006;64(8):43-8. 